\title{
A NOVEL COHERENT DIGITAL SWEEP OSCILLATOR WITH EXTREMELY LOW SWEEP RATES AND HARMONIC DISTORTION
}

\author{
M. M. AL-IBRAHIM (JARRAH)* \\ Etisalat College of Engtneering, Sharjah, \\ P.O. Box 980, United Arab Emirates
}

(Received 30 July 2000; In final form 12 September 2000)

A novel technique to efficiently increase the resolution of coherent digital sweep oscillators based on look-up-table (LUT) methods is proposed. The increase in resolution measured in terms of sweep rates is achieved while maintaining very low levels of spurious harmonic distortion. The proposed technique increases the LUT length to a level at which the spurious harmonic distortion is negligible. The proposed technique is based on partitioning the address register into three sets and dividing the available LUT length into five smaller tables addressed according to the content of the three address register sets. The proposed technique is simulated and its performance is compared with that of the known sweep oscillators. The simulation results show that the proposed technique is superior to all sweep oscillators reported in the literature.

Keywords: Harmonic distortion; Sweep rate; Digital sweep oscillators; Look-up-table; Fractional addressing; Interpolation methods

\section{INTRODUCTION}

Coherent digital sweep oscillators in which both the frequency and phase of the sweep signal are specified for all time are essential elements in many applications. They are used for target velocity estimation [1], time-delay spectrometry for calibration of ultrasonic

"Dr. Al-Ibrahim is with Etisalat College of Engineering on leave from Electrical Engineering Department, Jordan University of Science and Technology, Irbid-Jordan. e-mail: mjarrah@ece.ac.ae 
transducers [2], phase coding of sweep signals in communication applications, system characterization, radar, sonar, acoustic digital imaging, and the determination of system response with network analyzers [3-5].

There are few methods to generate coherent sweep signals. Coherent sweep signals can be generated by storing the samples of a predetermined sweep signal in a high speed digital memory and then reading the samples at appropriate time intervals. The limitation of this method is the time-bandwidth product. Other methods are limited by the maximum attainable frequency.

A new method for the generation of digital sweep signals was proposed by Pedersen [5]. His approach is based on real time digital evaluation of the phase of the desired sweep signal and then reading its value from a LUT. The total phase of the sweep signal is quadratic and, therefore, two integrators connected in series must be used to generate the total phase. The oscillator was implemented using a TTL logic circuit for which the sweep rate is determined by the parallel shifter on the address lines. By shifting the value of the total phase $p$ bits to the left, the least significant $p$ bits are discarded and the next $K$ bits of the accumulator are used as an address pointer to the LUT.

Pedersen sweep oscillator has sweep rates that can be varied in steps of two only and both its start frequency and start phase can be preset with integer values only. Moreover, its main disadvantage is the high level of spurious harmonic distortion due to the fact that the least significant $p$ bits are discarded [6-8]. A sweep oscillator was reported in [9] in which fractional addressing was utilized to reduce the level of spurious harmonic distortion. In fact the sweep oscillator in [9] exhibits the advantage of fine sweep rates as compared with that of Pedersen oscillator. Another sweep oscillator in which fractional addressing is utilized was reported in [10]. The major disadvantage of the digital sweep oscillator in [10] is that it requires the implementation of two independent Pedersen sweep oscillators and then evaluating the phase difference between the two generated sweep signals. The hardware requirements, therefore, make its implementation costly.

In this paper, we propose a novel technique to efficiently achieve extremely low sweep rates and at the same time reduce the spurious harmonic distortion associated with fractional addressing of the LUT. The proposed technique has the advantage of increased sweep 
resolution and reduced spurious harmonic distortion over all sweep oscillators reported in the literature and its hardware implementation is much simpler than the sweep oscillator in [10]. The proposed technique, $P 1$, is based on partitioning the address register bits into three sets, namely the most significant $N_{I}$ bits, the next $N_{F 1}$ bits, and finally the least significant $N_{F 2}$ bits. The contents of the $N_{I}, N_{F 1}$, and $N_{F 2}$ bits are used to address five separate LUTs as described in the sequel shortly. The resulting effective LUT length is $2^{N_{t}+N_{m 1}+N_{m 2}}$ while the actually needed LUT length is $2^{N_{1}}+2^{N_{m 1}+1}+2^{N_{m 2}+1}$.

In Section 2, we present a review of the relevant sweep oscillators. In Section 3, the proposed technique is presented and analyzed. Simulation results are presented and discussed in Section 4. Finally, the paper is concluded in Section 5.

\section{REVIEW OF PREVIOUS SWEEP OSCTLLATORS}

Coherent sweep signals can be generated [5] by performing real-time evaluation of the phase of a linearly swept signal, then the extraction of $\bmod 2 \pi$ from the total phase, and the generation of sine or cosine swept signals by means of LUT.

The frequency of a linear sweep signal increases linearly with time, as given by

$$
f(t)=S t+f_{0}
$$

where $S$ is the sweep rate in $\mathrm{Hz} / \mathrm{s}$, and $f_{0}$ is the start frequency in $\mathrm{Hz}$. The phase of a linearly swept signal is obtained by integrating $f(t)$, to obtain

$$
\phi(t)=\pi S t^{2}+2 \pi f_{0} t+\phi_{0}
$$

where $\phi_{0}$ is the initial phase. The sweep signal is given by

$$
s(t)=A \cos \left(\pi S t^{2}+2 \pi f_{0} t+\phi_{0}\right)
$$

By carrying out a digital integration twice using a counter and an accumulator, the quadratic phase function is obtained. The desired initial frequency and phase are achieved by presetting the counter and the accumulator. The mod $2 \pi$ is extracted and used as address for a 
sine LUT. The extraction of mod $2 \pi$ is very simple and is achieved by ignoring the least significant $p$ bits and considering the next $K$ bits of the accumulator to address a LUT of length $L=2^{K}$. It is shown in [5] that the sweep rate, initial frequency, and initial phase of Pedersen sweep oscillator are given by

$$
\begin{gathered}
S=\frac{1}{T_{c}^{2} 2^{(p+K)}} \quad H z / s \\
f_{0}=\frac{C O_{0}}{T_{c} 2^{(p+K)}} \quad H z \\
\phi_{0}=\frac{2 \pi A C_{0}}{2(p+K)} \quad \mathrm{rad}
\end{gathered}
$$

Where $T_{c}$ is the clock interval and $C O_{0}, A C_{0}$ are the initial contents of the counter and accumulator, respectively. Pedersen sweep oscillator suffers from two sources of quantization errors which degrade the quality of the generated sweep signals. Namely, the quantization of the sine wave sample values stored in the LUT due to finite word length limitations. This source of error produces a harmonic distortion that is determined by the type of number representation and number of bits, $b$, used to represent the value of a sample in the memory. For two's complement number representation with rounding, the level of harmonic distortion is approximately $2^{-2 b} / 6$ which can be brought to any desired level by increasing $b$. The second major source of error is due to the truncation of the address register content as a consequence of ignoring the least significant $p$ bits. Let the content of the address register at a specific instant of time be $n d=I+F$ where $I$ and $F$ are the integer and fractional parts of $n d$, respectively. The direct method of Pedersen simply considers $I$ and neglects $F$ in addressing the LUT. For $\Delta \theta=2 \pi / 2^{k}$, the actually read sample is $\sin [\Delta \theta \eta$ instead of $\sin [\Delta \theta(I+F)]$ which introduces a temporal quantization error that depends on $\Delta \theta, I$, and $F$ as given by

$$
e(\Delta \theta, I, F)=\sin [\Delta \theta I]-\sin [\Delta \theta(I+F)]
$$

The error sequence $e(\Delta \theta, I, F)$ was analyzed in $[7,8]$ and shown that it reduces the signal-to-noise ratio of a sinusoidal signal to a value in the range $\left[20 \log \left(2^{K}\right)-5.17,20 \log \left(2^{K}\right)-4.92\right] \mathrm{dB}$. It can be shown that the 
error sequence $e(\Delta \theta I, F)$ is an additive noise that causes a harmonic distortion approximately equal to $\Delta \theta^{2} / 12$. To compare between the two distortion sources, let the samples in the LUT be represented by 15 bits and let the LUT length $L\left(L=2^{K}\right)$ be $L=2048$. It follows that the word length constraint (15 bits) causes a level of harmonic distortion approximately equal to $\mathbf{- 9 8} \mathrm{dB}$ while the temporal quantization error, $e(\Delta \theta, I, F)$, results in a distortion of $-55 \mathrm{~dB}$. To avoid the distortion due to temporal quantization error, $e(\Delta \theta, I, F)$, $L$ must be greater than $2^{17}=131072$ which is obviously a formidable memory size. It should be noted that for a specified LUT length, the sweep rate of Pedersen sweep oscillator can be set at any desired extremely small value by simple adjustment of $p$ at the price of increased harmonic distortion. Consequently, interpolation methods [9] were used to reduce the amount of spurious harmonic distortion while maintaining low sweep rates or equivalently large effective LUT length.

Recently, a sweep oscillator that utilises two independent digital sweep oscillators that are identical to Pedersen oscillator was reported in [10]. Both sweep oscillators in [10] employ the trigonometric interpolation method presented in [6]. The trigonometric interpolation method can be summarized as follows:

$$
\sin [\Delta \theta(I+F)]=\sin [\Delta \theta I] \cos [\Delta \theta F]+\cos [\Delta \theta I] \sin [\Delta \theta F]
$$

The implementation of Eq. (8) requires two LUTs in addition to the original LUT which is of length $L$. In particular, we must have two more LUTs each of length $2^{N /}$ for storing the sample values of $\sin [\Delta \theta F]$ and $\cos [\Delta \theta F]$. It was shown in [6] that the level of harmonic distortion associated with trigonometric interpolation method is essentially the same as that of the direct LUT method with a table length $2^{N_{F}} L$. The first sweep oscillator in [10] utilises two LUTs each of length $L_{1}\left(L_{1}=2^{k}-1\right)$ for the storage of $\sin \left[\Delta \theta I_{1}\right]$ and $\cos \left[\Delta \theta I_{1}\right]$ and two LUTs each of length $2^{N_{F 1}}$ for the storage of $\sin \left[\Delta \theta F_{1}\right]$ and $\cos \left[\Delta \theta F_{1}\right]$. The second sweep oscillator in [10] utilises one LUT of length $L_{2}\left(L_{2}=2^{K}\right)$ for the storage of $\sin \left[\Delta \theta I_{2}\right]$ and $\cos \left[\Delta \theta I_{2}\right]$ and two LUTs each of length $2^{N_{r 2}}$ for the storage of $\sin \left[\Delta \theta F_{2}\right]$ and $\cos \left[\Delta \theta F_{2}\right]$. The sweep signals of the ith sweep oscillator are given by

$$
\begin{aligned}
& s_{i s}(t)=\sin \left(\pi S_{i} t^{2}+2 \pi f_{0 i} t+\phi_{0 i}\right) \quad i=1,2 \\
& s_{i c}(t)=\cos \left(\pi S_{i} t^{2}+2 \pi f_{0 i} t+\phi_{0 i}\right)
\end{aligned}
$$


Where $S_{t}, f_{0}$, and $\phi_{0 t}$ are given by

$$
\begin{gathered}
S_{i}=\frac{1}{T_{c}^{2} 2^{p_{i} L_{i} 2^{N_{F i}}}} \quad \mathrm{~Hz} / \mathrm{s} \\
f_{0 i}=\frac{C O_{0 i}}{T_{c} 2^{p_{i} L_{i} 2^{N_{F i}}}} \quad \mathrm{~Hz} \\
\phi_{0 i}=\frac{2 \pi A C_{0 i}}{2^{p_{i} L_{i} 2^{N_{F i}}}} \quad \mathrm{rad}
\end{gathered}
$$

The final desired sweep signal is given by

$$
\begin{aligned}
s_{o}(t) & =s_{1 s}(t) s_{2 c}(t)-s_{1 c}(t) s_{2 s}(t) \\
& =\sin \left[\pi\left(s_{1}-s_{2}\right) t^{2}+2 \pi\left(f_{01}-f_{02}\right) t+\phi_{01}-\phi_{02}\right]
\end{aligned}
$$

Each of the terms $s_{1 s}(t), s_{1 c}(t), s_{2 s}(t)$, and $s_{2 c}(t)$ in Eq. (13) requires two multipliers and an adder for its generation. Moreover, the generation of $s_{o}(t)$ from $s_{1 s}(t), s_{1 c}(t), s_{2 s}(t)$, and $s_{2 c}(t)$ requires two more multipliers and an adder. Therefore the implementation of Eq. (13) requires ten multipliers and five adders. One more problem not addressed in the design is the implementation of $\bmod 2 \pi$ for the sweep oscillator with $L_{1}=2^{k}-1$ which requires a digital circuit to be incorporated with the oscillator. The sweep rates associated with Eq. (13) for $p_{1}=p_{2}=p$, $L_{1}=2^{K}-1, L_{2}=2^{K}$, and $N_{F 1}=N_{F 2}=N_{F}$ is given by

$$
S_{o}=\frac{1}{T_{c}^{2} 2^{p} L_{1} L_{2} 2^{N_{F}}} \quad H z / s
$$

The effective LUT length associated with the sweep rates in Eq. (14) is $L_{1} L_{2} 2^{N_{F}}$. The spurious harmonic distortion due to temporal quantization errors is determined by the effective LUT length of the sweep oscillator and is in the order of $\left\{20 \log \left(L_{1} L_{2} 2^{N_{F}}\right)-5\right\} \mathrm{dB}[7,8]$. It should be noted that the level of spurious harmonic distortion of $s_{o}(t)$ is always higher than its predicted value as a result of the number of used multipliers. The proposed method for increasing the sweep resolution and at the same time maintaining extremely low levels of spurious harmonic distortion is described in the next section. 


\section{THE PROPOSED SWEEP OSCILLATOR}

In this section, we present a new technique to extremely increase the resolution of the sweep oscillator while maintaining a reduced harmonic distortion and hardware complexity. The proposed sweep oscillator utilises one Pedersen oscillator along with the technique $P 1$ for handling the content of the address register. The technique, $P 1$, is based on considering the fractional bits of the address register as three groups of $N_{F 1}, N_{F 2}$, and $p$ bits, respectively. The content of the least significant sweep controlling $p$ bits is discarded throughout the paper. For the remaining content, the content of the $N_{F 2}$ bits is the least significant while the content of the integer $N_{I}$ bits is the most significant.

Let the content of the address register $\left(N_{I}, N_{F 1}\right.$, and $N_{F 2}$ bits) at a specific instant of time be $n d=I+F_{1}+F_{2}$ where $I, F_{1}$, and $F_{2}$ are the integer and fractional parts of $n d$ respectively. Hence, the value of $\sin (\Delta \theta n d)$ is given by

$$
\begin{aligned}
\sin \left[\Delta \theta\left(I+F_{1}+F_{2}\right)\right]= & \sin \left[\Delta \theta\left(I+F_{1}\right)\right] \cos \left[\Delta \theta F_{2}\right] \\
& +\cos \left[\Delta \theta\left(I+F_{1}\right)\right] \sin \left[\Delta \theta F_{2}\right]
\end{aligned}
$$

The implementation of (15) requires the generation of $\sin \left[\Delta \theta\left(I+F_{1}\right)\right]$ and $\cos \left[\Delta \theta\left(I+F_{1}\right)\right]$ as described in [6] and is illustrated by the block diagram of Figure 1. Two more multiplier and an adder are needed to generate $\sin \left[\Delta \theta\left(I+F_{1}+F_{2}\right)\right]$ from $\sin \left[\Delta \theta\left(I+F_{1}\right)\right]$ and $\cos \left[\Delta \theta\left(I+F_{1}\right)\right]$. The generation of $\sin \left[\Delta \theta\left(I+F_{1}\right)\right]$ and $\cos \left[\Delta \theta\left(I+F_{1}\right)\right]$ is specified by

$$
\sin \left[\Delta \theta\left(I+F_{1}\right)\right]=\sin (\Delta \theta I) \cos \left(\Delta \theta F_{1}\right)+\cos (\Delta \theta I) \sin \left(\Delta \theta F_{1}\right)
$$

and

$$
\cos \left[\Delta \theta\left(I+F_{1}\right)\right]=\cos (\Delta \theta I) \cos \left(\Delta \theta F_{1}\right)-\sin (\Delta \theta I) \sin \left(\Delta \theta F_{1}\right)
$$

respectively. Equations (15-17) specify $P 1$ which requires one LUT of length $2^{N_{I}}$ for the storage of $\sin (\Delta \theta I)$ and $\cos (\Delta \theta I)$, two LUTs of length $2^{N_{F 1}}$ for the storage of $\sin \left(\Delta \theta F_{1}\right)$ and $\cos \left(\Delta \theta F_{1}\right)$, and two LUTs of length $2^{N_{F_{2}}}$ for the storage of $\sin \left(\Delta \theta F_{2}\right)$ and $\cos \left(\Delta \theta F_{2}\right)$. The generation of each of $\sin \left[\Delta \theta\left(I+F_{1}\right)\right]$ and $\cos \left[\Delta \theta\left(I+F_{1}\right)\right]$ require two multipliers and an adder which make the total number of multipliers 


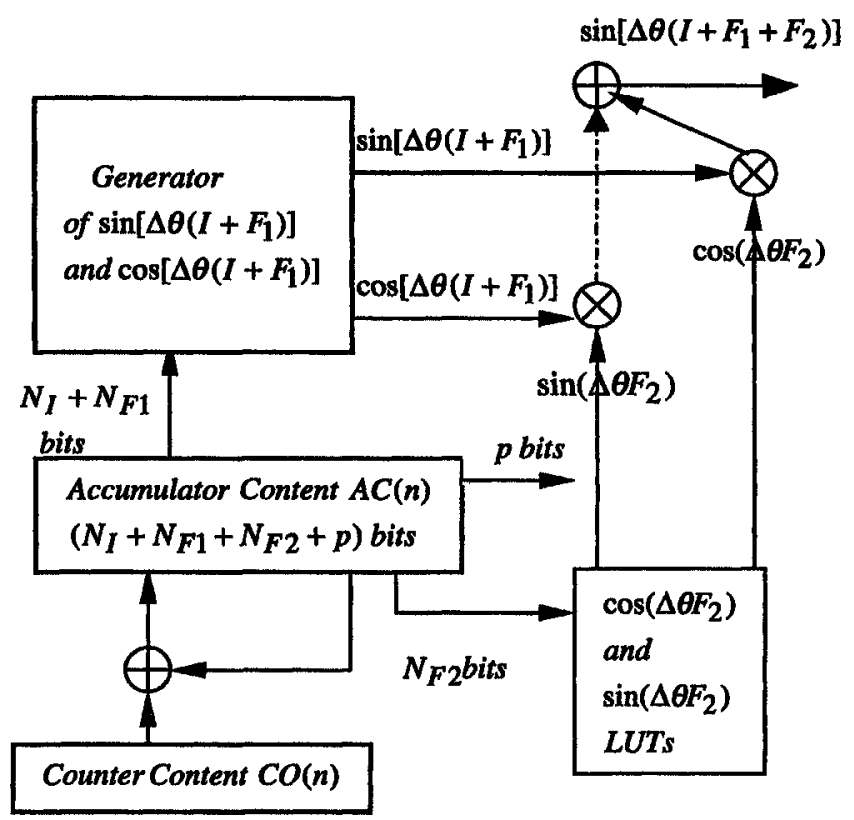

FIGURE 1 Block diagram of the first proposed sweep oscillator.

equal to six and the total number of adders equal to three. It should be noted that the sweep oscillator in [10] utilises two Pedersen sweep oscillators and requires ten multipliers and five adders. Besides, the proposed sweep oscillator requires five LUTs while the sweep oscillator in [10] requires seven LUTs for their hardware implementation.

Let the initial content of the counter at $n=0$ be $C O_{0}$ and the initial content of the accumulator be $A C_{0}$. It follows that the accumulator content after $n$ iterations is given

$$
A C(n)=A C_{0}+n C O_{0}+\frac{n(n+1)}{2}
$$

The angle (phase) $\phi(n)$ is obtained by extracting $\bmod 2 \pi$ from $A C(n)$ as given by

$\phi(n)=\bmod 2 \pi[A C(n)]=\frac{2 \pi}{2^{\left(N_{1}+N_{F 1}+N_{F 2}+p\right)}}\left[A C_{0}+n C O_{0}+\frac{n(n+1)}{2}\right]$ 
The frequency at time $t=n T_{c}$ is given by

$$
\begin{aligned}
f\left(n T_{c}\right) & =\frac{\phi(n)-\phi(n-1)}{2 \pi T_{c}} \\
& =\frac{1}{T_{c} 2^{\left(N_{t}+N_{p 1}+N_{F_{2}}+p\right)}}\left[C O_{0}+n\right]
\end{aligned}
$$

Similarly, the sweep rate at $t=n T_{c}$ is given by

$$
S\left(n T_{c}\right)=\frac{f\left(n T_{c}\right)-f\left((n-1) T_{c}\right)}{T_{c}}=\frac{1}{T_{c}^{2} 2^{\left(N_{t}+N_{F 1}+N_{m 2}+p\right)}}
$$

It is clear that the sweep rate in Eq. (21) is less than that of the oscillator in [10] and is significantly less than that of Pedersen oscillator. To compare the sweep rates of the three oscillators, let us assume that they all utilise a total memory of size 256 words. In this case, the sweep rate of Pedersen oscillator is $1 / T_{c}^{2} 2^{(8+p)} \mathrm{Hz} / \mathrm{s}$, while the sweep rate of the oscillator in [10] is $1 / T_{c}^{2} 2^{(10+p)} .31 \mathrm{~Hz} / \mathrm{s}$ and the sweep rate of the proposed oscillator is $1 / T_{c}^{2} 2^{(17+p)} \mathrm{Hz} / \mathrm{s}$.

Table I illustrates the saving in total LUT length achieved by using the proposed scheme over that used in [10] for some typical values of LUT lengths. It should be noted that the sweep oscillator in [10] uses one LUT of length $L_{2}$, two LUTs of length $L_{1}$ each, two LUTs of length $L_{2 F}$ each, and two LUTs of length $L_{1 F}\left(L_{1 F}=L_{2 F}=L_{F}\right)$ each. The effective LUT length of the oscillator in [10] is $L_{e 10}=L_{1} L_{2} L_{F}$ and requires a total LUT length $L_{T 10}=L_{2}+2 L_{1}+2 L_{2 F}+2 L_{1 F}$. The proposed oscillator uses one LUT of length $L_{l}$, two LUTs of length $L_{F 1}$ each, two LUTs of length $L_{F 2}$ each, and its effective LUT length is $L_{e p}=L_{T} L_{F 1} L_{F 2}$. It is evident from Table I that the proposed sweep oscillator utilizes a more efficient technique than that used in [10]. In fact, $L_{e p}$ is more than twice $L_{e 10}$, requires less total LUT length $\left(L_{1}+2 L_{F 1}+2 L_{F 2}=L_{T p}<L_{T 10}\right)$.

It is worth mentioning that for an available total memory of size 256 words, it follows that the difference between $\cos \left(\Delta \theta F_{2}\right)$ and 1 is in the order of $2^{-16}$ which allows us to discard the LUT containing the sample values of $\cos \left(\Delta \theta F_{2}\right)$. The number of required LUTs is four each of length 64 with an effective LUT length $L_{e p}=2^{18}$. Therefore, the implementation of the sweep oscillator requires two multipliers and an adder for the generation of each of $\sin \left[\Delta \theta\left(I+F_{1}\right)\right]$ and 


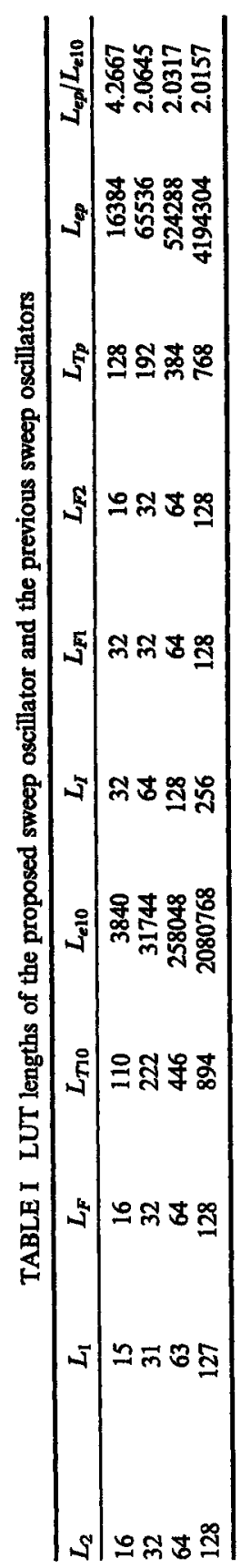


$\cos \left[\Delta \theta\left(I+F_{1}\right)\right]$. One more multiplier is required to implement the second term in Eq. (15) which make the total number of multipliers equal to five and the total number of adders equal to three.

\subsection{Bit Width Requirements}

Assuming that all sweep signals start from zero initial frequency, the lowest sweep rate, $S_{\min , P}$, of Pedersen sweep oscillator is given by [5]

$$
S_{\min , P}=f_{c l k}^{2} / 2^{n_{a}}
$$

where $f_{c l k}=1 / T_{c}$ is the clock frequency and $n_{a}$ is the bit width used for implementing the accumulator. It is clear that $n_{a}$ is determined by the maximum shift, $p_{\max }$ and is equal to $p_{\max }+K$.

Similarly, the frequency of Pedersen sweep oscillator at $t=n T_{c}$ is given by

$$
f(n)=\frac{f_{c l k}}{2^{p+K}} n
$$

Let the maximum allowed frequency, $f_{\max }$, be equal to $f_{c l k} / 8$, i.e., minimum sampling rate is 4 times higher than the Nyquist rate. It follows that the counter bit width, $n_{c}$, must satisfy the equation

$$
\frac{2^{n_{e}}}{2^{p_{\max }+K}}=\frac{2^{n_{c}}}{2^{n_{a}}}=\frac{1}{8}
$$

Solving Eq. (24) for $n_{c}$, we obtain $n_{c}=n_{a}-3$ bits.

The minimum sweep rate of the oscillator in [10] is given by

$$
S_{\min , 10}=f_{c l k}^{2} /\left(L_{1} 2^{n_{a}}\right)
$$

which shows an improvement factor of $L_{1}$ as compared with $S_{\min , p}$.

For the proposed sweep oscillator, the same analyses are valid after replacing the LUT length $2^{K}$ by the effective LUT length $L_{e p}$. Therefore, it follows that the lowest sweep rate of the proposed sweep oscillator, $S_{\min , p}$, is given by

$$
S_{\min , p}=f_{c l k}^{2} /\left(L_{e p} 2^{P_{\max }}\right)
$$


To this end, let $K=8$ bits, then for the available LUT length, $L_{1}=31\left(L_{e 10} \cong 2^{15}\right)$, and $L_{e p}=2^{17}$. Therefore,

$$
S_{\min , p}=f_{c l k}^{2} /\left(2^{9} 2^{K+P_{\max }}\right)
$$

Equation (27) shows that $S_{\min , p}$ is $2^{-9}$ times lower than $S_{\min , P}$ and $2^{-4}$ times lower than $S_{\min , 10}$. The time-bandwidth product of the proposed sweep oscillator is, therefore, $2^{9}$ times higher than that of Pedersen and $2^{4}$ times higher than that of the sweep oscillator in [10].

\subsection{Performance Calculations}

The performance of the proposed sweep oscillator is compared with that of Pedersen and the sweep oscillator in [10] assuming the same LUT lengths. For the sweep oscillator in [10] let $L_{1}=255$ and $L_{2}=256\left(L_{e 10} \cong 2^{16}\right)$, it follows that the LUT for Pedersen oscillator is 768 and for the proposed oscillator is $L_{I}=256, L_{F 1}=128$, and $L_{F 2}=128\left(L_{e p}=2^{22}\right)$. Let the clock frequency be fixed at $f_{c l k}=80 \mathrm{MHz}$.

Let us assume that the sweep time of Pedersen sweep oscillator be $100 \mathrm{~s}$ from zero initial frequency to $f_{\max }=10 \mathrm{MHz}$. It follows that $S_{\min , P}=10^{5} \mathrm{~Hz} / \mathrm{s}$ with $n_{a}=36$ bits, $n_{c}=33$ bits, and $p_{\max }=26$ bits. For the same sweep time, the sweep oscillator in [10] requires two accumulators with $n_{a}=28$ bits each, two counters with $n_{c}=25$ bits each. The proposed sweep oscillator requires one accumulator with $n_{a}=36$ bits, one counter with $n_{c}=33$ bits, and $p=14$ bits.

The minimum sweep time is obtained by setting $p=0$. In this case, the sweep rates are maxima, $S_{\max }$. Clearly, $S_{\max , P}=8.333 \times 10^{12} \mathrm{~Hz} / \mathrm{s}$ and the sweep time is only $1.2 \mu \mathrm{s}$ while $S_{\max , 10}=9.8039 \times 10^{10} \mathrm{~Hz} / \mathrm{s}$ with a sweep time equal to $120 \mu \mathrm{s}$. For the proposed sweep oscillator, $S_{\max , P}=1.5259 \times 10^{9} \mathrm{~Hz} / \mathrm{s}$ with a sweep time equal to $6553.6 \mu \mathrm{s}$.

The maximum sweep time is obtained by setting $p=26$ bits. In this case, the sweep rates are minima, $S_{\text {min }}$. The sweep rate of Pedersen, $S_{\min , P}=9.313 \times 10^{4} \mathrm{~Hz} / \mathrm{s}$ and the sweep time is $107.37 \mathrm{~s}$. For the same value of $p, n_{a}=36$ bits, and $n_{c}=33$ bits, it follows that $S_{\min , 10}=365.224 \mathrm{~Hz} / \mathrm{s}$ and the sweep time is $7.606 \mathrm{hr}$. For the proposed sweep oscillator, $S_{\min , p}=22.737 \mathrm{~Hz} / \mathrm{s}$ and the sweep time is $122.168 \mathrm{hr}$. This is certainly a direct consequence of the efficient increase in the LUT length. 


\section{SIMULATION RESULTS}

The Pedersen sweep oscillator, the oscillator in [10], and the proposed oscillator are all simulated using a MATLAB program. The simulation results are obtained assuming a sixteen-bit word length for all arithmetic operations. The program also evaluates the samples of the ideal system. The spectra of the ideal and all other generated samples are also evaluated assuming that the system uses $f_{c l k}=80 M H z$. We assume that Pedersen sweep oscillator uses a LUT of length $L=4096$ ( $K=12$ bits). However, the proposed sweep oscillator and the sweep oscillator in [10] are allowed to use a total LUT of length less than or equal to 128. For the sweep oscillator in [10], $L_{1}=15, L_{2}=16, L_{F}=16\left(L_{T 10}=110\right.$ and $\left.L_{e 10}=3840\right)$. The parameters of the proposed sweep oscillator are $L_{I}=32, L_{F 1}=32$, $L_{F 2}=16\left(L_{T p}=128\right.$ and $\left.L_{e p}=16384\right)$. All sweep oscillators are simulated in the same sweep range. Figures 2,3 show the output spectra of Pedersen sweep oscillator with $p=0$ and the ideal sweep oscillator. The oscillator in [10] and the proposed oscillator have

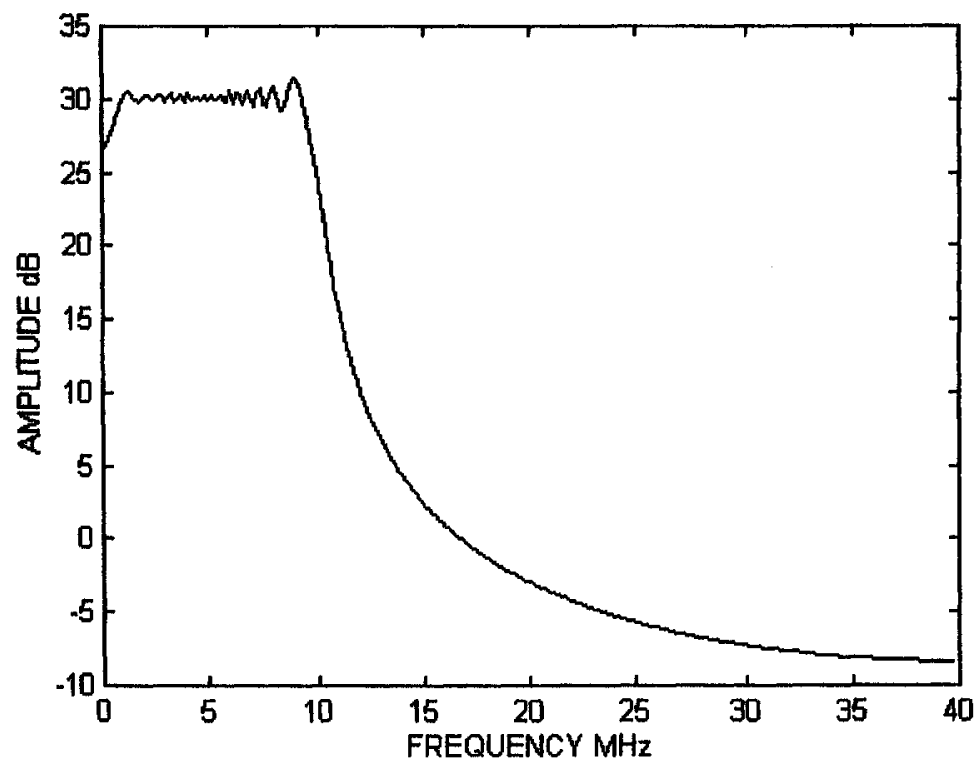

FIGURE 2 Amplitude spectrum of Pedersen sweep oscillator with $p=0$. 


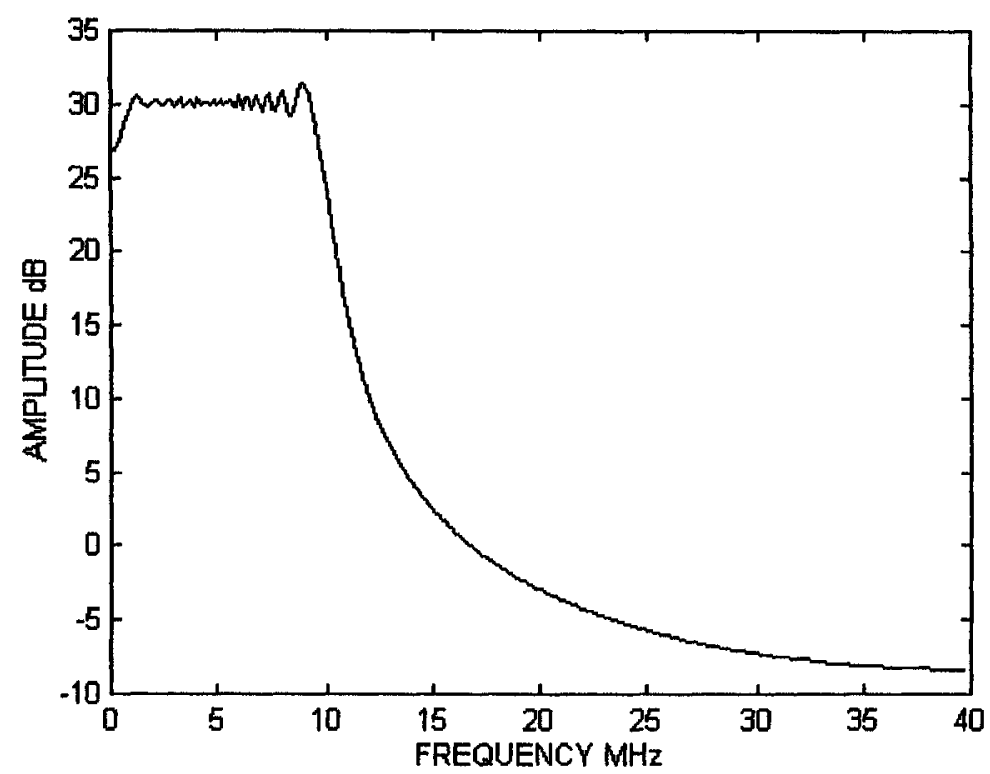

FIGURE 3 Amplitude spectrum of ideal sweep oscillator with $p=0$.

amplitude spectra which can not be distinguished from the spectrum of the ideal sweep oscillator when $p=0$. However, in order to actually evaluate the performance, the amplitude spectra of the error sequence, defined as the difference between the output of the sweep oscillator and that of the ideal sweep oscillator, are plotted for various sweep oscillators in Figures 4-6. It is obvious from Figures 4-6 that the level of spurious harmonic distortion associated with Pedersen sweep oscillator is less than its counterpart for other sweep oscillators and that the level of spurious harmonic distortion associated with the oscillator in [10] is the highest. This is obviously due to the repeated quantization of the multipliers outputs.

Figures 7-10 show the spectra for Pedersen oscillator with $p=4$, the ideal sweep oscillator with $p=4$, the oscillator in [10] with $p=4$, and the proposed oscillator with $p=2$. This case is significant because all sweep oscillators are subject to temporal quantization errors. It is evident from Figures 7,9 that the amplitude spectrum of Pedersen sweep oscillator is almost identical to that of the oscillator in [10]. Figures 11-13 provide a closer look by plotting the amplitude spectra of the error sequences associated with the various sweep oscillators. 


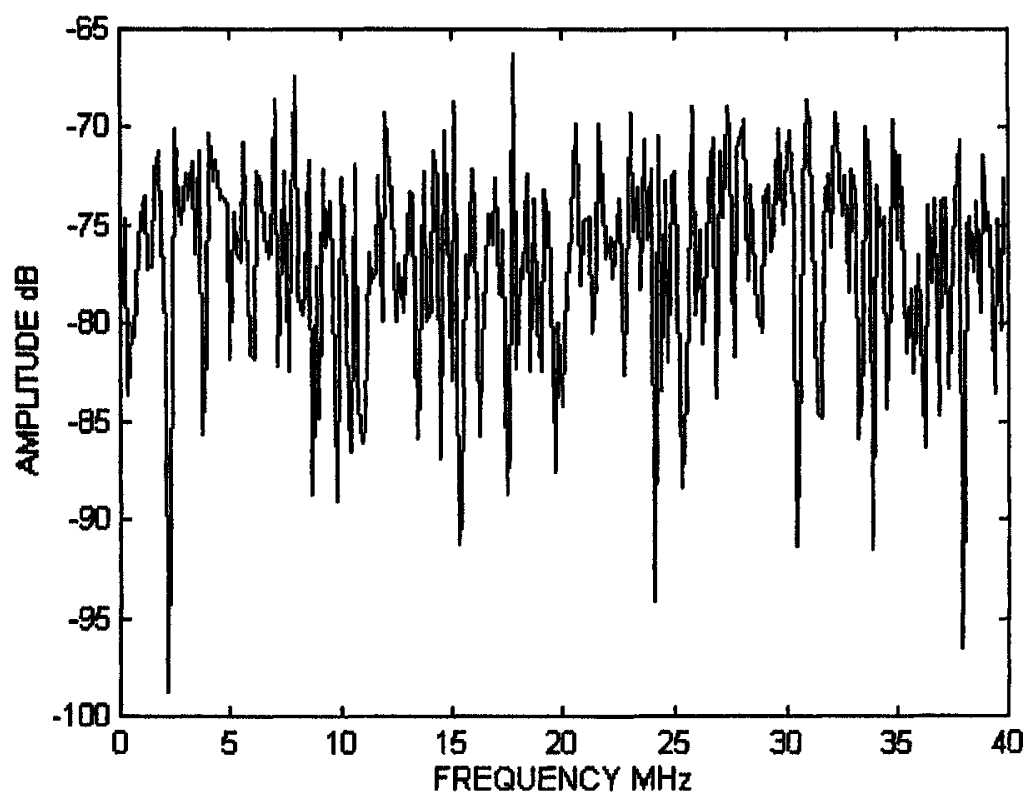

FIGURE 4 Error spectrum of Pedersen sweep oscillator with $p=0$.

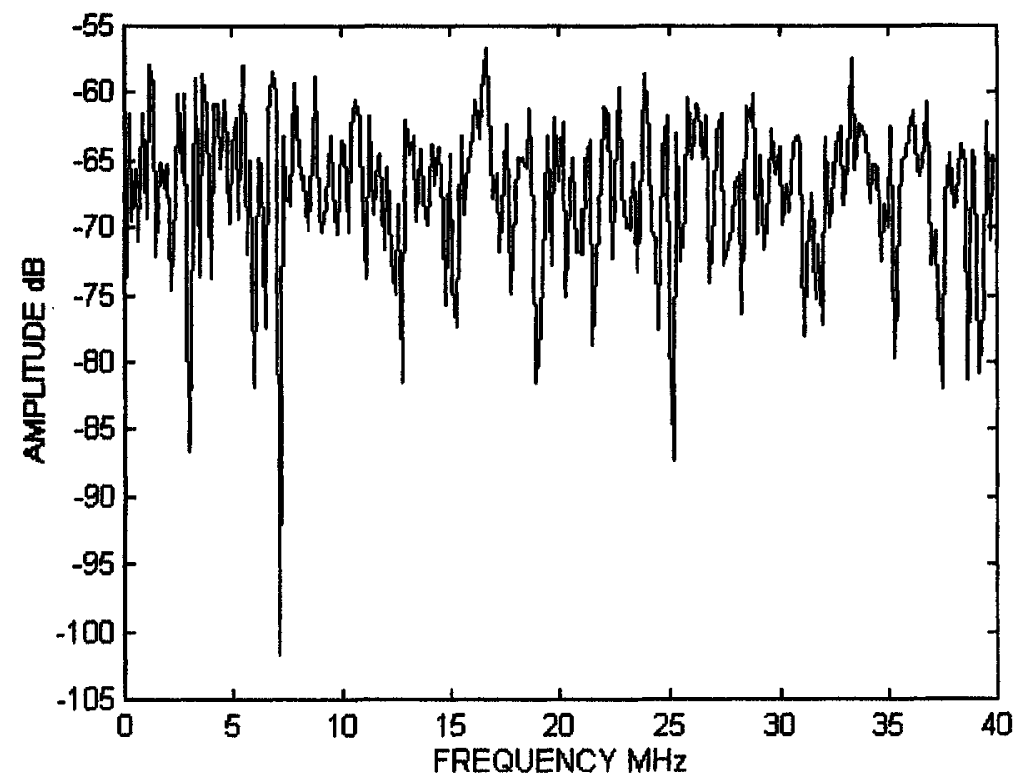

FIGURE 5 Error spectrum of the oscillator in [10] with $p=0$. 


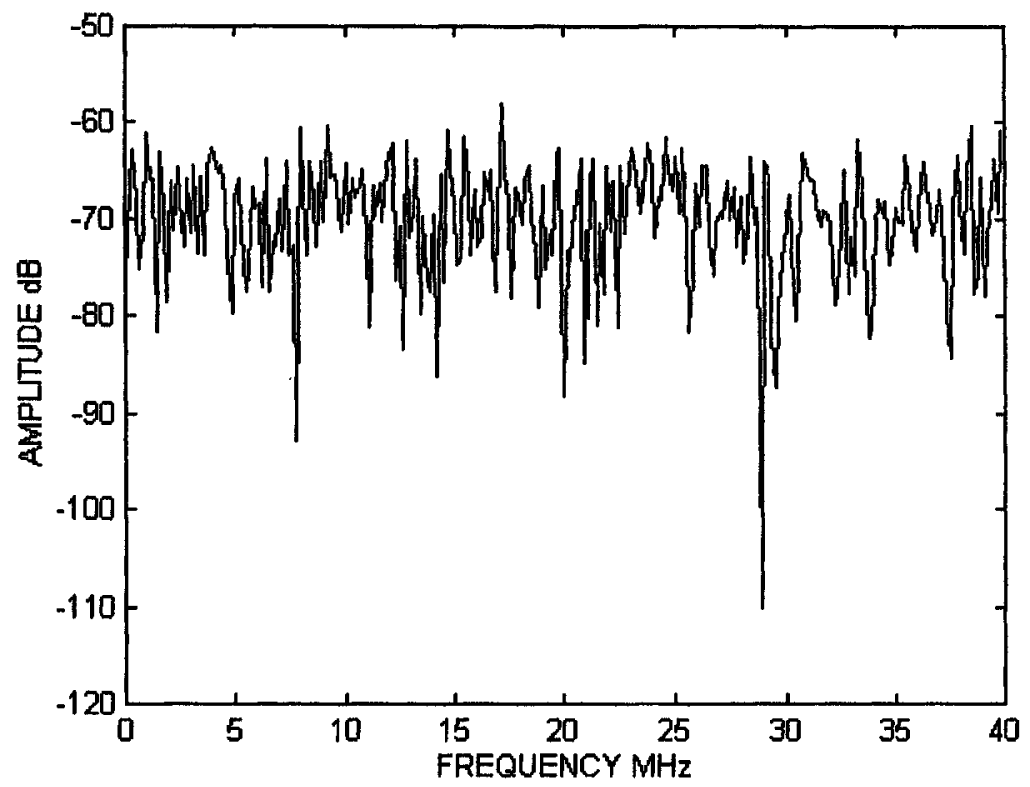

FIGURE 6 Error spectrum of the proposed sweep oscillator with $p=0$.

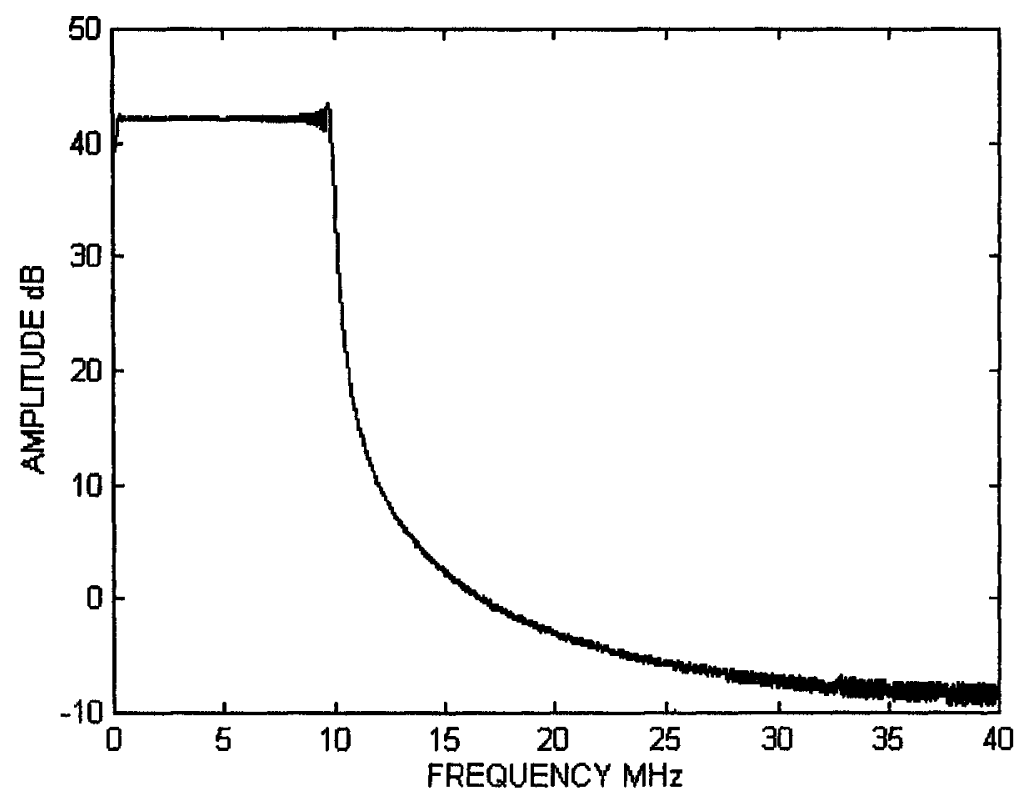

FIGURE 7 Amplitude spectrum of Pedersen sweep oscillator with $p=4$. 


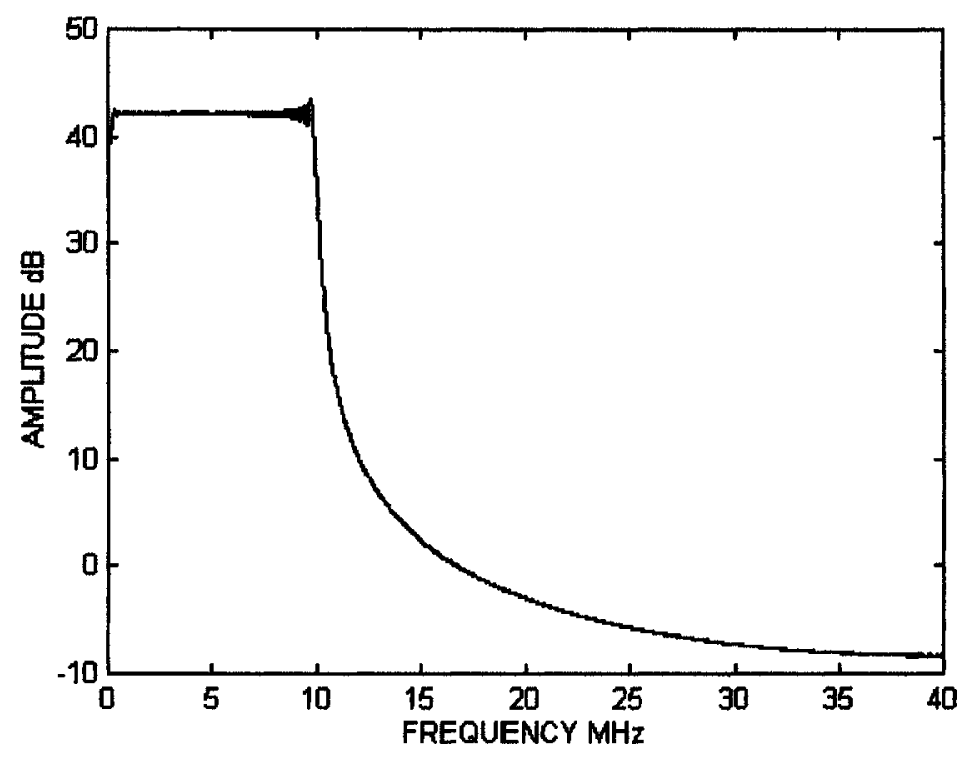

FIGURE 8 Amplitude spectrum of ideal sweep oscillator with $p=4$.

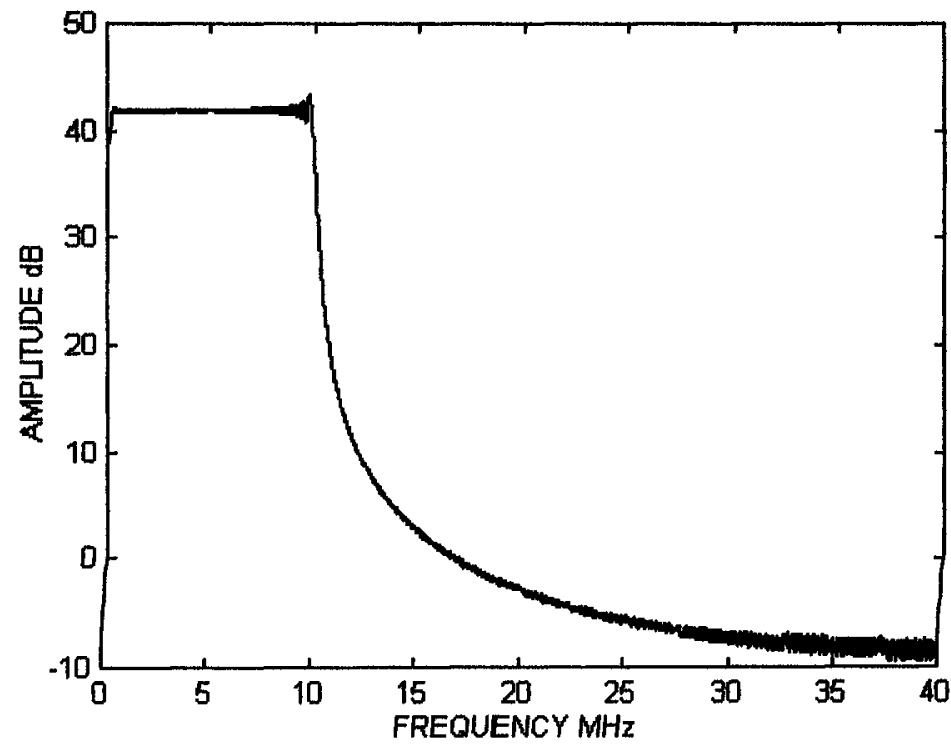

FIGURE 9 Amplitude spectrum of the sweep oscillator in [10] with $p=4$. 


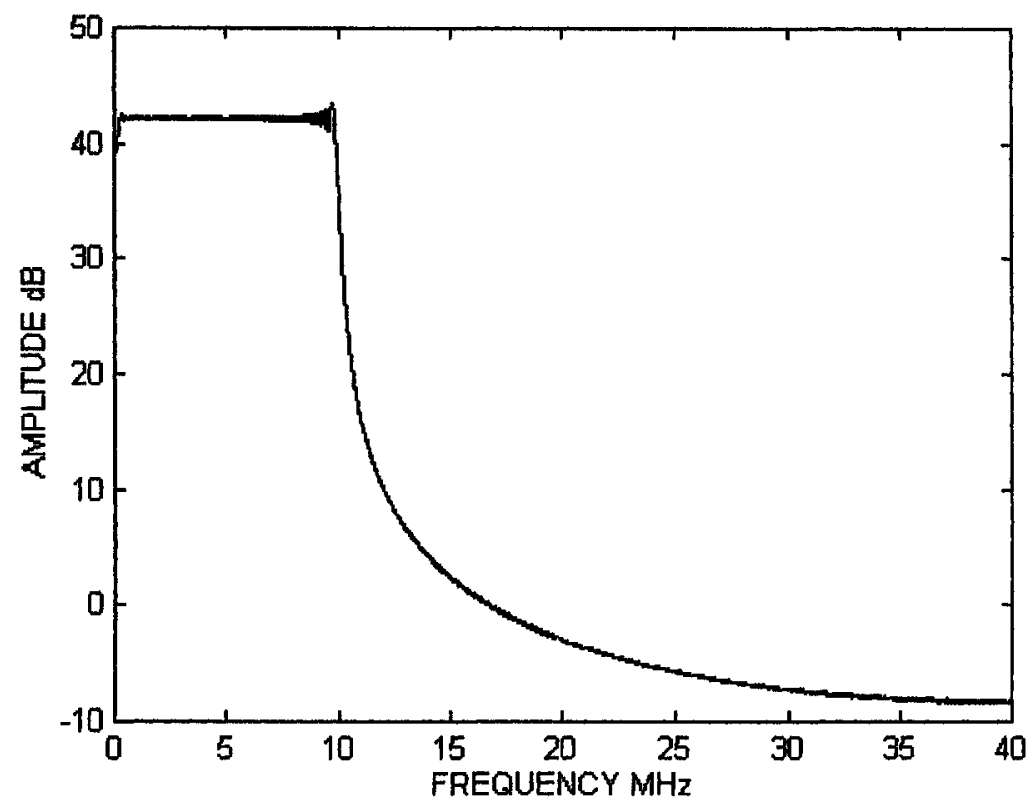

FIGURE 10 Amplitude spectrum of the proposed sweep oscillator with $p=2$.

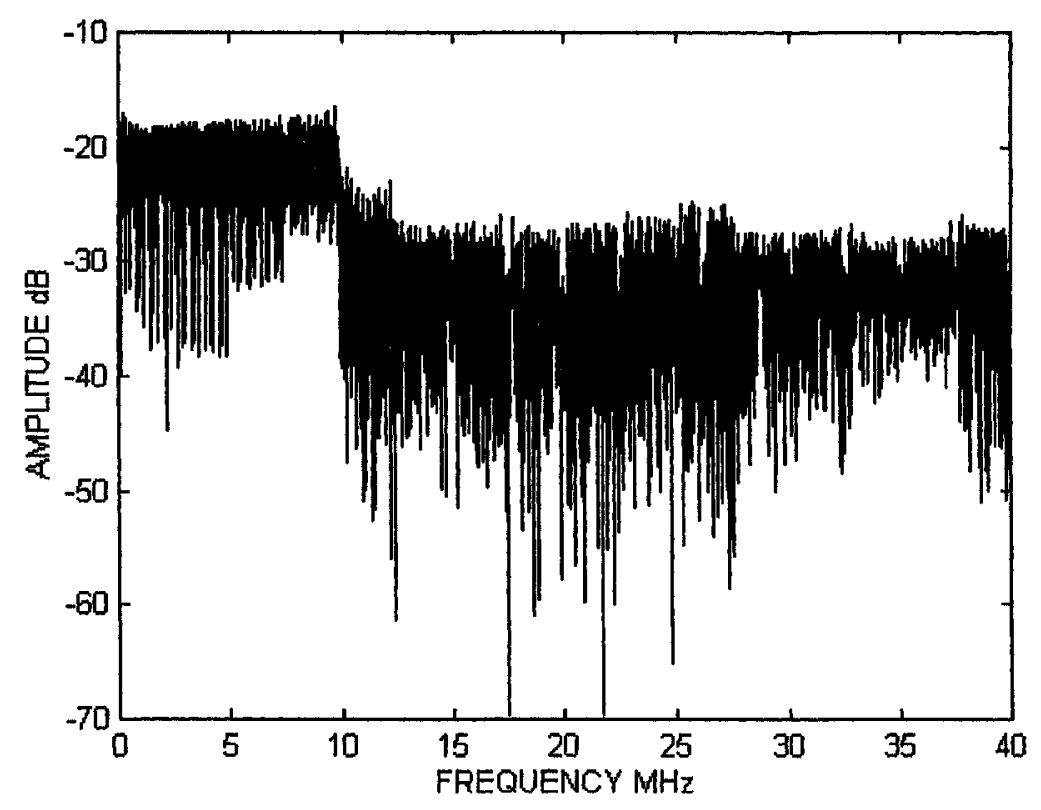

FIGURE 11 Error spectrum of Pedersen sweep oscillator with $p=4$. 


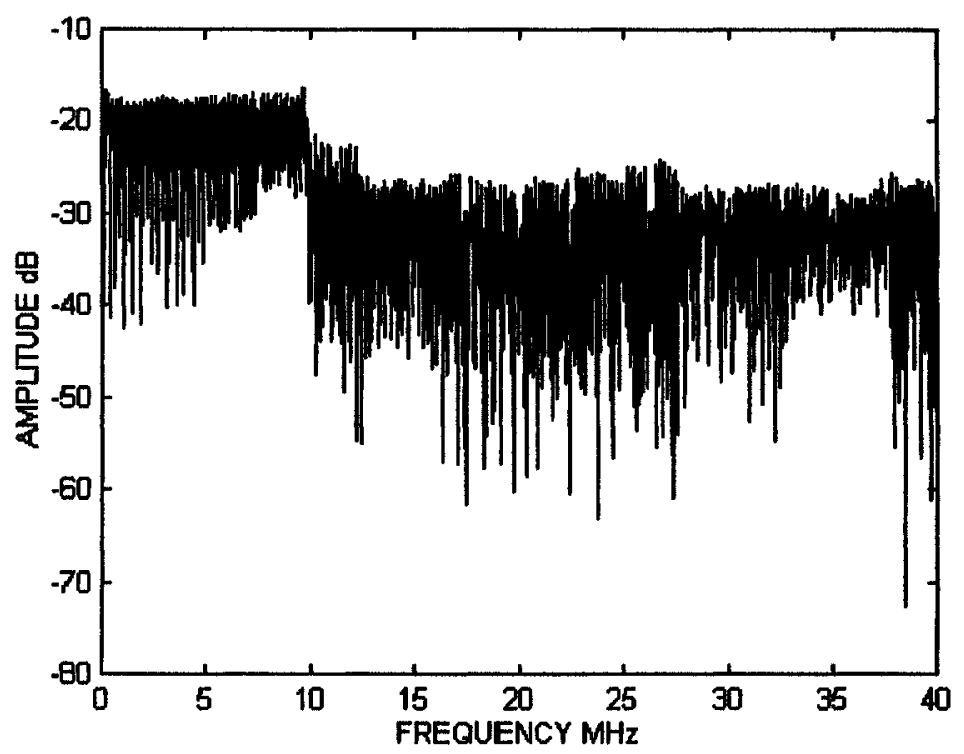

FIGURE 12 Error spectrum of the sweep oscillator in [10] with $p=4$.

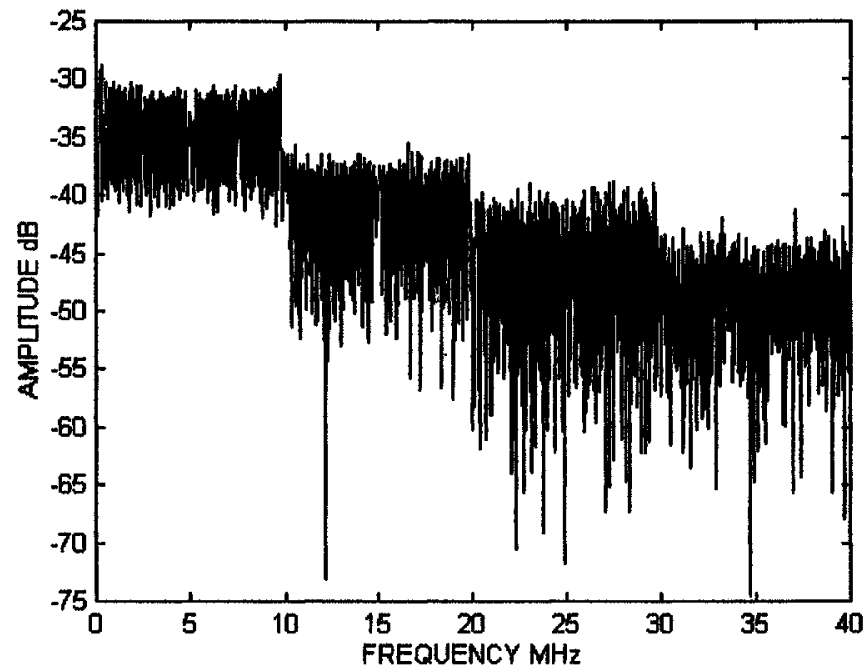

FIGURE 13 Error spectrum of the proposed sweep oscillator with $p=2$. 
It is evident from Figures 11-13 that the performance of the sweep oscillator in [10]is less than that of Pedersen sweep oscillator as a consequence of the number of multipliers used. The proposed sweep oscillator has an error spectrum level higher than its level for the case $p=0$. The performance degradation due to temporal quantization errors of the proposed sweep oscillator is smaller than that of the other oscillators and is in the range predicted by theory.

\section{CONCLUSION}

In this paper, a novel technique is proposed to significantly increase the sweep resolution by achieving extremely low sweep rates without increasing the amount of spurious harmonic distortion. The proposed sweep oscillator utilizes the available total LUT size in a manner which is the most efficient known in the literature. In fact, with a LUT of length equal to 256 , we can achieve any desired sweep rate. The timebandwidth product of the proposed sweep oscillator is superior to that of any coherent digital sweep oscillator reported in the literature. The simulation results show that the spurious harmonic distortion of the proposed sweep oscillator is significantly less than that of both Pedersen sweep oscillator and the sweep oscillator in [10]. The proposed sweep oscillator is suitable for implementation on already available digital signal processors like TMS32010 DSP or it can be fabricated on a single chip using monolithic large-scale integration.

\section{References}

[1] Wilhjelm, J. E. and Pederson, P. C. (1993). "Target velocity estimation with FM and PW echo ranging Doppler systems-Part II: system analysis", IEEE Trans. Ultrason. Ferroelectr. Freq. Control, 40, 373-378.

[2] Pederson, P. C., Lewin, P. A. and Jorono, L. B. (1988). “Application of time-delay spectrometry for calibration of ultrasonic transducers", IEEE Trans. Ultrason. Ferroelectr. Freq. Control, 35, 185-205.

[3] Plumb, R. G. and Ma, H. (1993). "Swept frequency reflectometer design for in-situ permitivity measurement", IEEE Trans. Instrum. Meas., 42, 730-734.

[4] Garcia, D., "Precision digital sine wave generation with the TMS32010", In: Digital Signal Processing Applications with the TMS32010 Family. Dallas, TX (Texas Instruments, 1986), pp. 269-289.

[5] Pederson, P. C. (1990). "Digital generation of coherent sweep signals", IEEE Trans. Instrum. Meas., 39, 90-95. 
[6] Schanerberger, M. and Awad, S. S. (1990). "The implementation of digital sine wave oscillator using the TMS320C25: Distortion reduction and applications", IEEE Trans. Instrum. Meas., 39, 870-873.

[7] Jeng, Y. C. (1988). "Digital spectra of nonuniformly sampled signals: Fundamentals and high-speed waveform digitizers", IEEE Trans. Instrum. Meas., 37, 245-251.

[8] Jeng, Y. C. (1988). “ Digital spectra of nonuniformly sampled signals: Digital lookup tunable sinusoidal oscillator", IEEE Trans. Instrum. Meas., 37, 358-362.

[9] Al-Ibrahim, M. M. and Al-Khateeb, A. M. (1997). "Improved digital sweep oscillator", Int. J. Electron., 83, 753-760.

[10] Hiasat, A. A. and Al-Khateeb, A. M. (1998). "Efficient digital sweep oscillator with extremely low sweep rates", IEE Proc. Cir., Dev., Syst., 145, 409-414. 

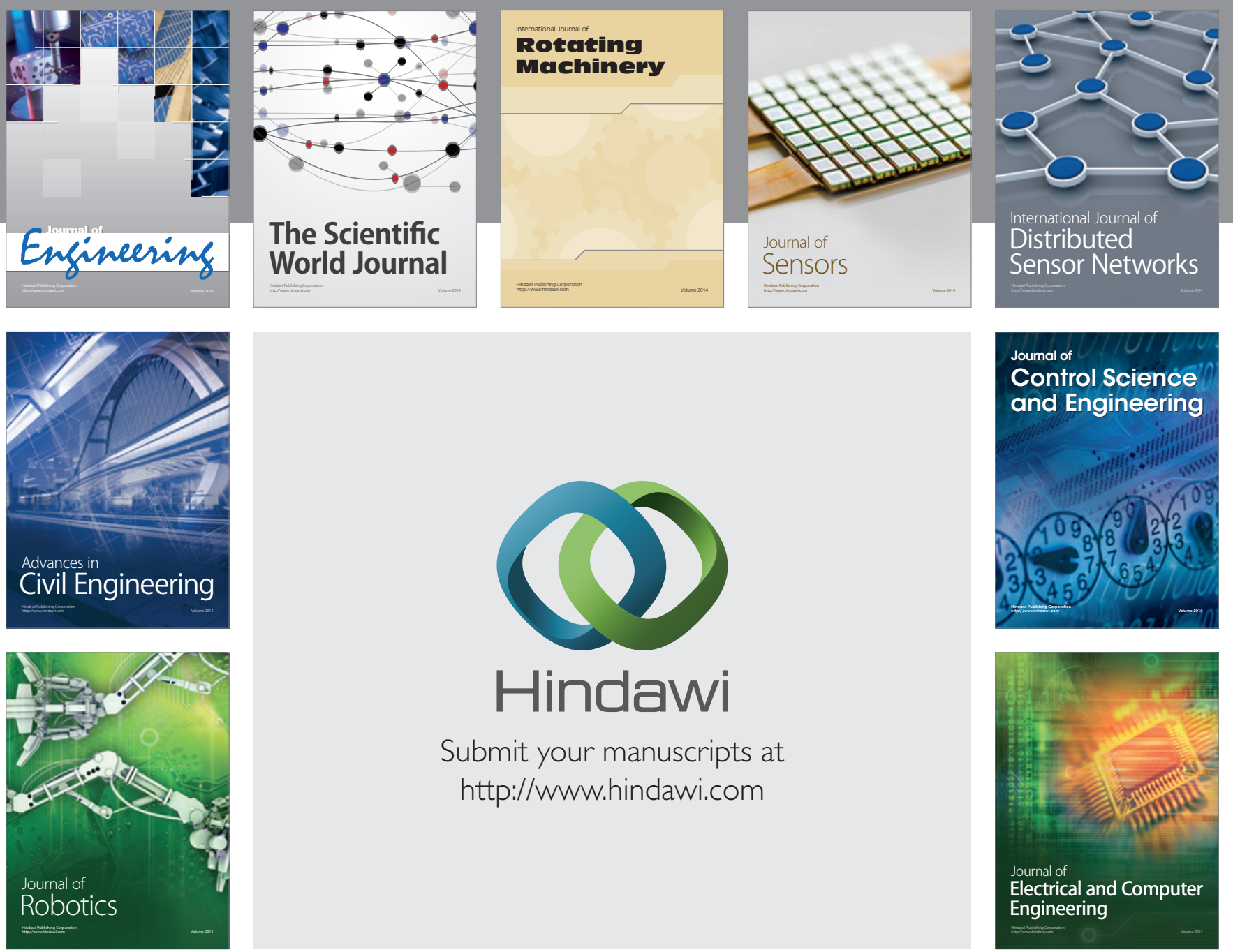

Submit your manuscripts at

http://www.hindawi.com
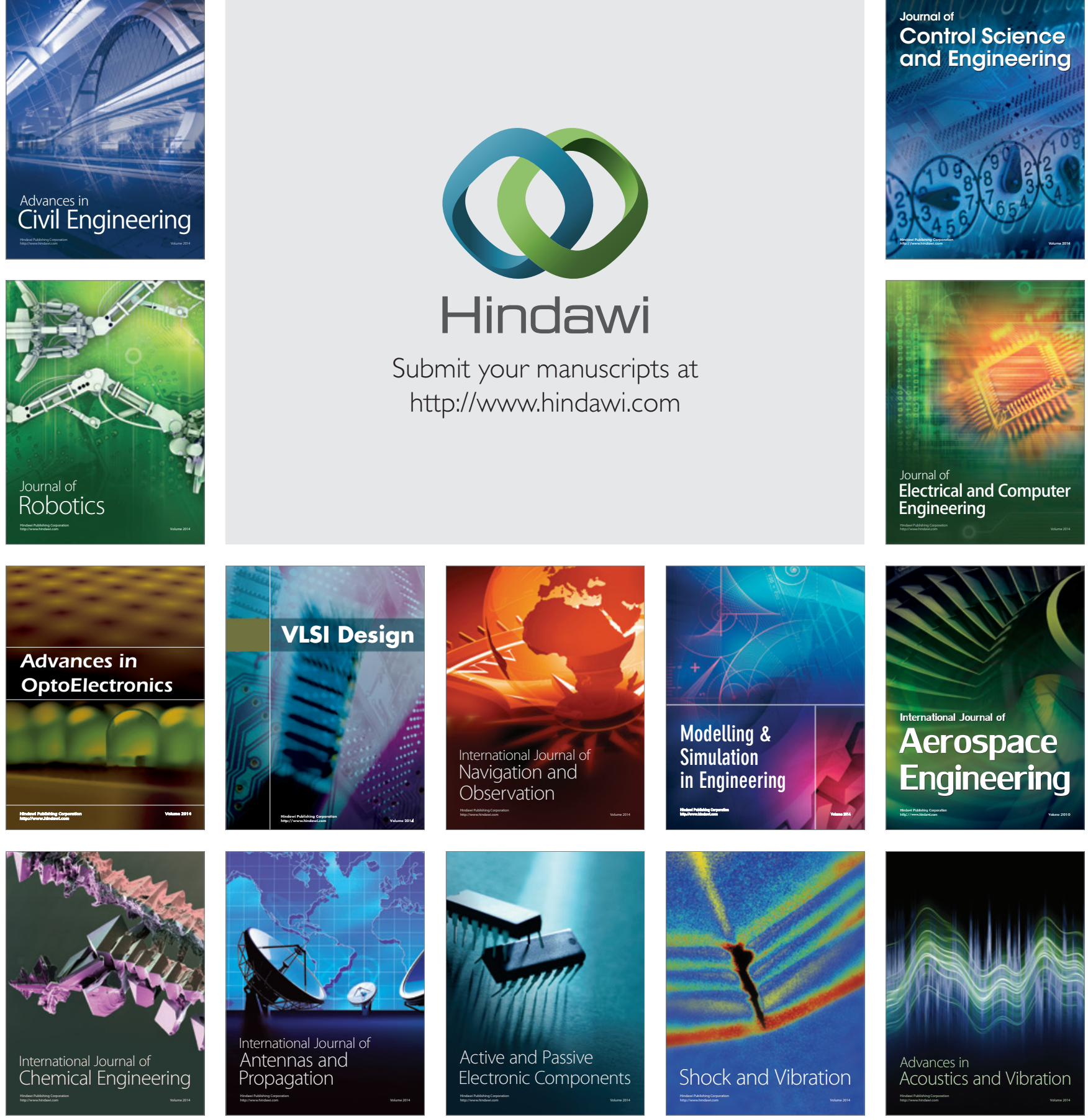